\title{
Editorials
}

\section{Safer 'chemsex':}

\section{GPs' role in harm reduction for emerging forms of recreational drug use}

\section{WHAT IS NEW IN RECREATIONAL DRUG USE?}

A rapidly changing pattern of drug use is emerging that requires health services to find new approaches to harm reduction. The widespread use of 'club drugs' has led to many serious harms that have attracted much media attention over the past few years.' 'Club drugs' are drugs such as methylenedioxy- $\mathrm{N}$-methylamphetamine (MDMA or ecstasy), methamphetamine, and ketamine that are used in a recreational fashion to enhance social experience, usually being taken at dance clubs, concerts, and parties.

The number of people needing treatment for club drugs has increased; club drug users in England now constitute $5 \%$ of all adult presentations for drug treatment and $14 \%$ of all presentations by those $<18$ years of age. ${ }^{2}$ Since 2008, the use of novel psychoactive substances (NPS) or 'legal highs' (drugs that are synthesised to mimic the effects of traditional recreational drugs; currently marketed as plant food or bath salts in the UK) has grown exponentially ${ }^{3}$ but the latest evidence suggests further trends in who is using drugs and how they are being taken. One such emerging subculture is that of 'chemsex' or 'party and play'. These terms refer to the use of recreational drugs immediately before and/or during sex to facilitate or enhance sexual pleasure.

\section{'CHEMSEX'}

The drugs most commonly associated with chemsex are methamphetamine (crystal meth), gamma-hydroxybutyric acid (GHB), gamma-butyrolactone (GBL), and methylmethcathinone (mephedrone). These drugs can induce euphoria, increased energy, and disinhibition but also enhance sexual arousal and aid sexual stamina. ${ }^{4}$ Other drugs such as ketamine and cocaine are used in chemsex but to a lesser extent. A combination of drugs can be used at the same time. Although such drugs can enhance sexual pleasure, there is also concern that chemsex is associated with particular harms including sexual risktaking behaviour. ${ }^{5}$

Chemsex seems to be a problem that particularly affects a small but significant subsection of men who have sex with men (MSM). However, taking recreational or prescription drugs before or during sex has been associated with high-risk sexual

"The drugs [associated with chemsex] have been associated with increased sexual risk behaviours, such as unprotected sex, which may lead to an increase in sexually transmitted infections (STIS), including HIV.

behaviours for both MSM and heterosexuals for over a decade ${ }^{6,7}$ and is not just confined to the UK. ${ }^{8,9}$ Evidence on the extent of chemsex, who it affects, and associated harms is still emerging. ${ }^{4}$ It is also difficult to distinguish when drugs are used to enhance or facilitate the sexual encounter, and whether it strictly excludes other drugs such as phosphodiesterase type 5 inhibitors and alkyl nitrates, and their associated harms.

\section{PROBLEMS ASSOCIATED WITH CHEMSEX}

Chemsex presents potentially harmful effects that are different from those linked to other recreational drugs and how they are used. Methamphetamine, GHB/GBL, and mephedrone can all lead to unwanted effects including agitation, anxiety, paranoia, aggression, and psychoses. Intense comedowns can cause users to feel suicidal. All drugs can lead to dependency for the user, which can happen quite rapidly after a relatively short, intense period of use. There are reports of overdose with unconsciousness and death. ${ }^{10}$ Serious drug interactions with a range of drugs including alcohol and HIV antiretroviral medications can occur.

The drugs have been associated with increased sexual risk behaviours, such as unprotected sex, which may lead to an increase in sexually transmitted infections (STIS), including HIV. The drugs may also increase disinhibition and hypersexuality, leading to unwanted sexual experiences that users later regret. Some drugs can induce coma and there are reports of use associated with sexual assault. ${ }^{4}$ The anaesthetic nature of some drugs facilitates longer sexual encounters, sex with multiple partners, and can lead to increased rectal, penile, or vaginal trauma, all of which may facilitate STI/HIV transmission. Some drugs can be administered intravenously, which increases the risk of transmission of blood- borne viruses including HIV and hepatitis B and $\mathrm{C}$. There are concerns about emerging patterns of injecting drug use, particularly among MSM, and there is evidence to suggest poorer adherence to antiretroviral medications and drug interactions among HIV-positive chemsex users. ${ }^{11,12}$

\section{WHAT CAN WE DO TO LIMIT THE HARMS?}

Individuals who engage in chemsex, just like users of club drugs, perceive themselves to be different from opiate and crack users, who have traditionally been the main clients of drug services and to whom GPs may be more familiar with managing. Chemsex users are more likely to be in employment and to have well-connected social networks. ${ }^{13}$ Chemsex users may feel that traditional drug services are not appropriate for their needs and may be more likely to present to general practice, yet clinicians in this setting may lack confidence and be unfamiliar with managing the harms associated with chemsex use or of the drug services available.

There is limited evidence of effective screening strategies for reducing the harms of chemsex. However, we use sexual history to make a risk assessment for STIs and HIV, and we often include questions about alcohol and drug use; the enquiry could logically lead to questions about drug use during sex. Secondary prevention can be done through sensitive enquiry especially in cases of sexual assault, apparent overdose, and in some cases of multiple episodes of STIs or new diagnoses of blood-borne viruses. We should use these opportunities to assess appropriateness of referral to drug services for harm minimisation.

\section{SEEKING HELP}

There are specialist drug clinics in London but help and support are available elsewhere (for example, i-access Surrey \& Borders Drug \& Alcohol Services: http://www. 
